\title{
Platypnoea-orthodeoxia syndrome, an underdiagnosed cause of hypoxaemia: four cases and the possible underlying mechanisms
}

\author{
M. Nassif $\cdot$ H. Lu $\cdot$ T.C. Konings $\cdot$ B.J. Bouma $\cdot$ A. Vonk Noordegraaf $\cdot$ B. Straver $\cdot$ N.A. Blom $\cdot$ \\ S.A. Clur · A.P.C.M. Backx · M. Groenink · S.M. Boekholdt · D.R. Koolbergen · M.G. Hazekamp · \\ B.J.M. Mulder · R.J. de Winter
}

Published online: 14 July 2015

(C) The Author(s) 2015. This article is published with open access at Springerlink.com

\begin{abstract}
Cardiac platypnoea-orthodeoxia syndrome (POS) is a position-dependent condition of dyspnoea and hypoxaemia due to right-to-left shunting. It often remains unrecognised in clinical practice, possibly because of its complex underlying pathophysiology. We present four consecutive patients with POS and patent foramen ovale (PFO) who underwent a successful percutaneous PFO closure, describe the mechanism of their POS and provide a review of the literature.
\end{abstract}

Keywords Platypnoea-orthodeoxia - Dyspnoea

Foramen ovale, patent $\cdot$ Atrial septum $\cdot$ Mechanism

\section{Introduction}

Platypnoea-orthodeoxia syndrome (POS) is an uncommon clinical condition, characterised by position-dependent dys-

R.J. de Winter $(\bowtie) \cdot$ M. Nassif $\cdot$ H. Lu $\cdot$ B.J. Bouma

B. Straver · N.A. Blom · A.P.C.M. Backx · M. Groenink ·

S.M. Boekholdt · B.J.M. Mulder

Department of Cardiology, Cardiac Catheterization Laboratory,

Academic Medical Center-University of Amsterdam,

PO Box 22660, 1100 DD Amsterdam, The Netherlands

e-mail: r.j.dewinter@amc.uva.nl

T.C. Konings

Department of Cardiology, VU University Medical Center,

Amsterdam, The Netherlands

A.V. Noordegraaf

Department of Pulmonary Medicine, VU University Medical

Center,

Amsterdam, The Netherlands

B. Straver · N.A. Blom · S.A. Clur · A.P.C.M. Backx Department of Pediatric Cardiology, Academic Medical Center, Amsterdam, The Netherlands pnoea and oxygen desaturation in an upright position which resolves by lying supine. In addition to cardiac defects, pulmonary and abdominal defects have also been reported in association with POS (Tables 1 and 2) [1]. POS was first described in a patient with an atrial septal defect (ASD); however, cardiac POS has subsequently been more often observed in patients with a patent foramen ovale (PFO) [2]. What seems essential for cardiac POS to exist are both an anatomical communication between the right and left atrium as well as a structural component that redirects shunt flow causing right-to-left shunting in the upright position [3]. Usually this structural component is a deformation of the right atrium or of the inter-atrial septum. POS is primarily characterised by normal right atrial and pulmonary artery pressures contrary to Eisenmenger's syndrome [4]. The exact mechanism of position-dependent change in shunting, including the gravitational forces on the cardiac structures involved, remains elusive. The final common

\author{
N.A. Blom \\ Department of Pediatric Cardiology, Leiden University Medical \\ Center, \\ Leiden, The Netherlands \\ D.R. Koolbergen · M.G. Hazekamp \\ Department of Cardiothoracic Surgery, Academic Medical \\ Center, \\ Amsterdam, The Netherlands \\ D.R. Koolbergen · M.G. Hazekamp \\ Department of Pediatric Cardiac Surgery, Leiden University \\ Medical Center, \\ Leiden, The Netherlands \\ B.J.M. Mulder \\ Interuniversity Cardiology Institute of the Netherlands (ICIN), \\ Utrecht, The Netherlands
}


Table 1 Conditions associated with cardiac POS

\begin{tabular}{ll}
$\begin{array}{l}\text { Group A: anatomic preferential } \\
\text { blood flow across the inter-atrial } \\
\text { communication }\end{array}$ & $\begin{array}{l}\text { Group B: transient reversal of } \\
\text { left-to right pressure gradient }\end{array}$ \\
\hline $\begin{array}{l}\text { Congenital abnormality } \\
\text { Absent superior vena cava }\end{array}$ & $\begin{array}{l}\text { Pulmonary } \\
\text { Chronic obstructive pulmo- } \\
\text { nary disease }\end{array}$ \\
Ascending aortic aneurysm & Pneumonectomy \\
Atrial septal aneurysm & Pulmonary embolism \\
Ebstein's anomaly & Pulmonary hypertension \\
Partial anomalous venous return & Cardiac \\
Persistent left superior vena cava & Constrictive pericarditis \\
Prominent Eustachian valve & Pericardial adipose deposition \\
Transposition of the great vessels & Pericardial effusion \\
Unroofed coronary sinus & \\
Post-surgical repair & \\
Aortic valve replacement & \\
Ascending aorta repair & \\
Atrial switch procedure & \\
Fontan procedure & \\
Tumours & \\
Cardiac cyst/mass & \\
Lipomatous hypertrophy of the \\
inter-atrial septum
\end{tabular}

Diseases associated with POS in case of inter-atrial communication. Conditions in group A cause POS through anatomical distortion of the right atrium (e.g. congenital abnormality, post-surgical repair, cardiac or hepatic cyst) or the atrial septum (e.g. a thin, floppy septum primum in lipomatous hypertrophy of the inter-atrial septum), or by disposition of the right ventricle (e.g. an inflamed, thick-walled ventricle due to eosinophilic endomyocardial disease) leading to preferential flow through the inter-atrial septum. In group B the pulmonary and cardiac conditions cause POS in the presence of a right-to-left pressure gradient due to pulmonary vascular resistance and compression of the right ventricular inflow tract respectively. Adapted from Knapper et al. [1].

pathway appears to be a position-dependent opening of the PFO with right-to-left shunting. We present four cases of POS treated at the Centre for Congenital Heart Disease Amsterdam Leiden (CAHAL), and discuss the pathophysiological mechanism of POS and its diagnostic and therapeutic pitfalls.

\section{Case 1}

A 46-year-old woman was referred to our hospital for percutaneous PFO closure, after having experienced symptoms of dyspnoea in the upright and left supine position for several months. She had a history of chronic obstructive pulmonary disease GOLD II and had undergone left pneumonectomy
Table 2 Non-cardiac conditions associated with platypnoea-orthodeoxia syndrome

\begin{tabular}{lll}
\hline Pulmonary & Abdominal & Other \\
\hline $\begin{array}{l}\text { Acute respiratory } \\
\text { distress syndrome }\end{array}$ & Paralytic ileus & Chest wall trauma \\
$\begin{array}{lll}\text { Chronic obstruc- } \\
\text { tive pulmonary }\end{array}$ & $\begin{array}{l}\text { Hepatopulmo- } \\
\text { nary syndrome }\end{array}$ & $\begin{array}{l}\text { Diabetic autonomic } \\
\text { neuropathy }\end{array}$
\end{tabular}

disease

Cryptogenic fibros-

ing alveolitis

Fat embolism

Hemidiaphragmat-

ic dysfunction

Pleural effusion

$\begin{array}{ll}\text { Alcoholic } & \text { Organophosphate } \\ \text { liver cirrhosis } & \text { poisoning } \\ \text { Autoimmune } & \text { Paraoesophageal hernia } \\ \text { hepatitits } & \text { repair } \\ \text { Hepatitis A } & \text { Parkinson's disease }\end{array}$

Hepatitis A Parkinson's disease

Noncir- Vertebral fractures

rhotic portal

hypertension

Pneumocystis and

Schistosomiasis Kyphoscoliosis

CMV pneumonia

Pneumonectomy

Pulmonary

arteriovenous

malformations

Pulmonary

embolism

Radiation-induced

bronchial stenosis

Traumatic bron-

chial rupture

Non-cardiac associations with POS include pulmonary conditions which cause pulmonary vascular shunting (e.g. arteriovenous malformation), ventilation-perfusion mismatching (e.g. pleural effusion, pneumonia, bronchial stenosis) or an anatomic distortion of the right atrium or ventricle (e.g. hemidiaphragmatic dysfunction). Hepatopulmonary syndrome causes POS through a high alveolararterial gradient (e.g. in portal hypertension, hepatitis). Orthostatic hypotension as a symptom of autonomic dysfunction (e.g. Parkinson, diabetic autonomic neuropathy, organophosphate poisoning) causes POS through an increase in orthostatic alveolar dead space and subsequent ventilation-perfusion mismatching. $C M V$ Cytomegalovirus.

6 months earlier, to remove an adenocystic carcinoma. In the weeks, thereafter, she developed progressive and position-dependent shortness of breath. Physical examination revealed a blood pressure of $100 / 65 \mathrm{mmHg}$ with a resting heart rate of 122 beats $/ \mathrm{min}$. She had central cyanosis, with a transcutaneous oxygen saturation of $91 \%$, while breathing $1.5 \mathrm{~L}$ oxygen as measured by pulse oximetry. Her heart sounds were shifted to the left on auscultation. Chest X-ray (Fig. 1a1) and computed tomography (CT) scan of the thorax showed a midline shift of the right lung with a consequent position of the heart to the left thoracic wall. No evidence was found of cancer recurrence or other pulmonary causes such as pleural effusion or atelectasis. Echocardiographic images revealed a PFO (Fig. 1a2) and a right-to-left shunt fraction measured $20 \%$ when breathing $100 \%$ oxygen in an upright position. At this point the patient was severely incapacitated; she could only lie on her right side, which 
had resulted in severe muscular atrophy. POS was finally diagnosed 6 months after the onset of her symptoms. Cardiac catheterisation was performed, which showed a normal right atrial and pulmonary artery pressure, and pulse oximetric saturations of $90 \%$ and $83 \%$ in right and left supine positions, respectively. The PFO was successfully closed with a $25 \mathrm{~mm}$ Amplatzer PFO Occluder (St. Jude, Minneapolis, USA) after which the arterial saturation improved immediately to $97 \%$ in the upright position. Her dyspnoea was resolved but an extensive rehabilitation course followed. She was able to gradually resume her daily routine and her exercise capacity improved significantly. No complications have been reported in 3 years of clinical followup. On her last transthoracic echocardiogram (TTE), the estimated systolic pulmonary artery pressure was $28 \mathrm{mmHg}$ and no residual shunt was seen over the closed PFO.

\section{Case 2}

A 36-year-old woman with a history of right mid and lower lobe resection due to a carcinoid in the right main bronchus 3 years earlier was referred to our hospital. She complained of progressive pain and dyspnoea since her operation, which was most prominent during exercise and when lying in the right supine position. Before the bilobectomy she had participated in competitive long distance running, but now the referring pulmonologist noted that she desaturated from 96 to $87 \%$ with severe dyspnoea after performing only 10 squats. Previously, a bronchoscopy revealed an open but narrow bronchus to the right upper lobe. This narrowing worsened in the right supine position and was therefore thought to be the cause of her dyspnoea in the right supine position. One year later, cardiac magnetic resonance (CMR) imaging and resting transoesophageal echocardiography (TEE) showed a PFO without a right-to-left shunt as measured in normal supine position so no POS was suspected (Fig. 1b1-2). None of the previous diagnostic tests could explain the coexistent position-dependent and exerciseinduced dyspnoea. A cardiopulmonary exercise test showed an early arterial partial $\mathrm{CO}_{2}$ pressure $\left(\mathrm{PaCO}_{2}\right)$ increase during exercise. Also, an increase of end-tidal $\mathrm{O}_{2}$ pressure and decreased end-tidal $\mathrm{CO}_{2}$ pressure were seen at an acute load of $150 \mathrm{~W}$, with a desaturation from 96 to $93 \%$ seen on pulse oximetry. Both her pulmonologist and cardiologist agreed that her symptoms had to be attributed to a mechanism involving her PFO. Firstly, the exercise-induced right-toleft shunt itself, and secondly, the consequently triggered ventilatory impairment related to the loss of alveolar tissue after pulmonary resection preventing adequate compensatory hyperventilation to normalise the $\mathrm{PaCO}_{2}$. Subsequently, the PFO was closed successfully with a Premere $25 \mathrm{~mm}$ device (St. Jude, Minneapolis, USA). She underwent exten- sive cardiac rehabilitation and on her latest outpatient visit, after 4 years of follow-up, no complications were found. She reported having run a marathon again, despite her unchanged ventilatory impairment. Her final TTE showed a normal pulmonary artery pressure and no residual shunt.

\section{Case 3}

A 73-year-old woman presented to our emergency department with acute dyspnoea and a severe hypoxaemia with a saturation of $63 \%$ measured by pulse oximetry in the upright position. She had a history of frequent recurrent pulmonary embolism due to essential thrombocytosis; however, a CT scan of the thorax excluded recurrent pulmonary embolism as a cause of dyspnoea this time. A TTE was performed to exclude pulmonary hypertension. Surprisingly, the echocardiogram in the left supine position revealed a PFO and a mildly elevated systolic pulmonary artery pressure of $36 \mathrm{mmHg}$. A subsequent TEE confirmed the existence of POS; pulse oximetric saturations dropped from $100 \%$ and $82 \%$ within $60 \mathrm{~s}$ when the patient changed from supine to upright position. Coincidentally, she was known with a stable aortic root and ascending aorta dilatation (diameters of $42 \mathrm{~mm}$ and $49 \mathrm{~mm}$ respectively, Fig. 1c1). The PFO was successfully closed with an Amplatzer $18 \mathrm{~mm}$ PFO Occluder. Due to a residual shunt on the procedural TEE, an additional $6 \mathrm{~mm}$ ASD Septal Occluder device was also implanted (Fig. 1c2) and the PFO was successfully closed. At one year follow-up she had no residual shunt and she had physically recovered from her former incapacitated condition.

\section{Case 4}

A 61-year-old male patient was referred to our hospital's congenital team for PFO closure due to POS. He had a history of pulmonary adenocarcinoma and had undergone a right pneumonectomy 6 months earlier. After the subsequent chemotherapy, he complained of progressive general deterioration and dyspnoea in the upright position. On presentation he was unable to walk more than five steps. Physical examination showed saturations of $97 \%$ versus $90 \%$ in the supine and upright position, respectively, measured by pulse oximetry with $3 \mathrm{~L}$ oxygen. Analysis by contrast echocardiography revealed a right-to-left shunt due to a PFO with a measured shunt fraction of $26 \%$. The chest X-ray showed a complete shift of the heart to the right (Fig. 1d1). CMR showed pleural effusion in the right hemithorax (Fig. 1d2) and no evidence was found for shunting in the supine position. During diagnostic heart catheterisation, systolic and diastolic pulmonary artery pressures were $33 \mathrm{mmHg}$ and 
Fig. 1 Different imaging modalities of case 1(a), 2 (b), 3 (c) and 4 (d), respectively, for the purpose of diagnosing POS by PFO. a1 Chest X-ray showing the heart position against the left thoracic wall. a2 Four chamber view of a Doppler TEE with a right-to-left shunt by PFO. b1 CMR showing malposition of the right thoracic wall resulting in a heart shift to the right and presence of PFO (arrow). b2 Short axis basal view of a Bubble contrast TEE showing no resting rightto-left shunting over the PFO. c1 CT angiography showing the dilated ascending aorta $(A)$ and aorta root $(B)$. c2 Periprocedural angiographic image of both Amplatzer devices. d1 Chest X-ray showing a tracheal shift to the left after pneumonectomy. d2 CMR shows a right hemithorax filled with pleural effusion and a compressed right atrium (arrow). $R A$ right atrium, $L A$ left atrium, $R V$ right ventricle, $L V$ left ventricle, $R$ right, $A O$ aorta, $P A$ pulmonary artery, $S V C$ superior vena cava
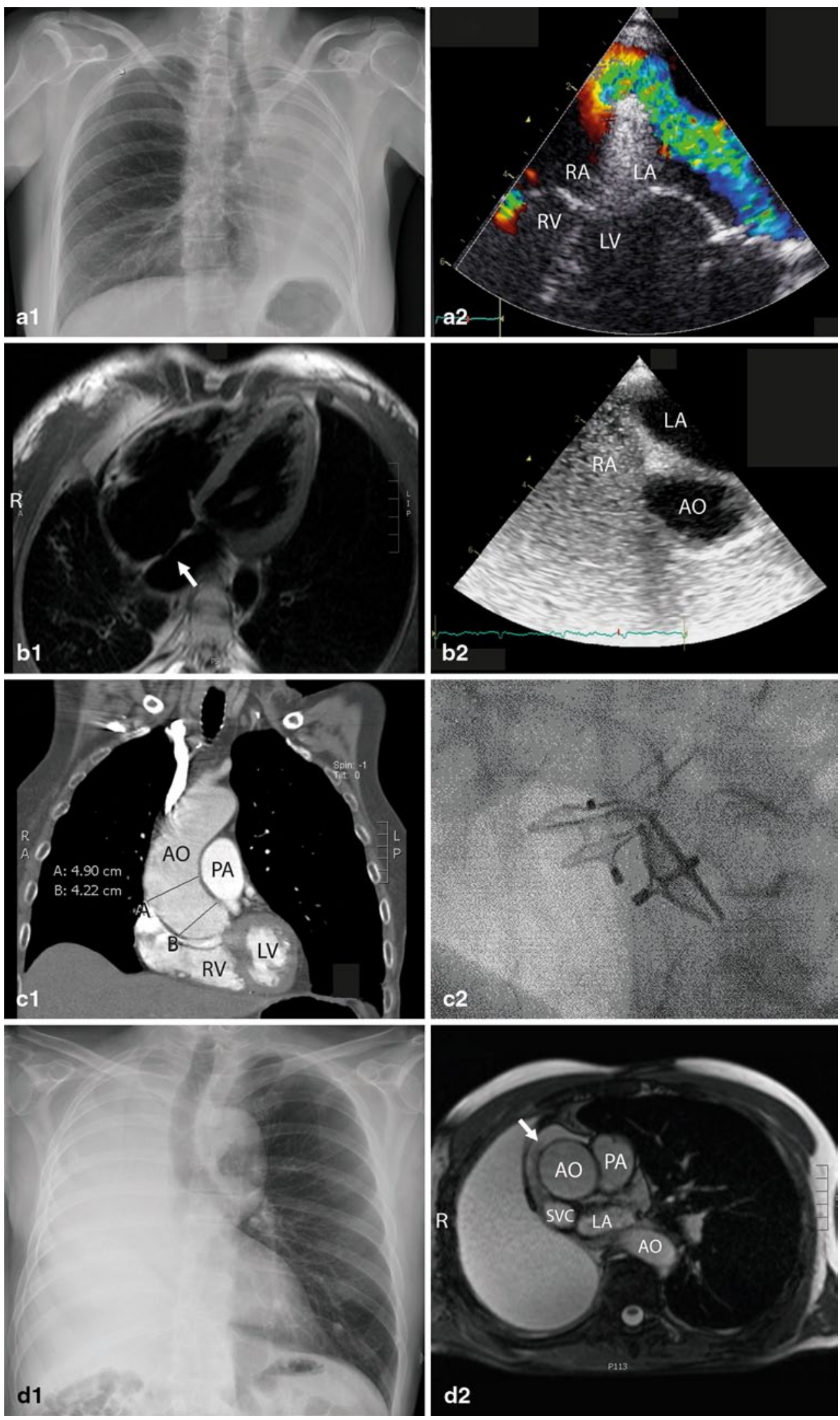

$16 \mathrm{mmHg}$, respectively, when lying supine, and $48 \mathrm{mmHg}$ and $28 \mathrm{mmHg}$, respectively, in the upright position. Arterial blood gas during catheterisation provided oxygen saturations of $96 \%$ and $85 \%$ in supine and upright position. Due 


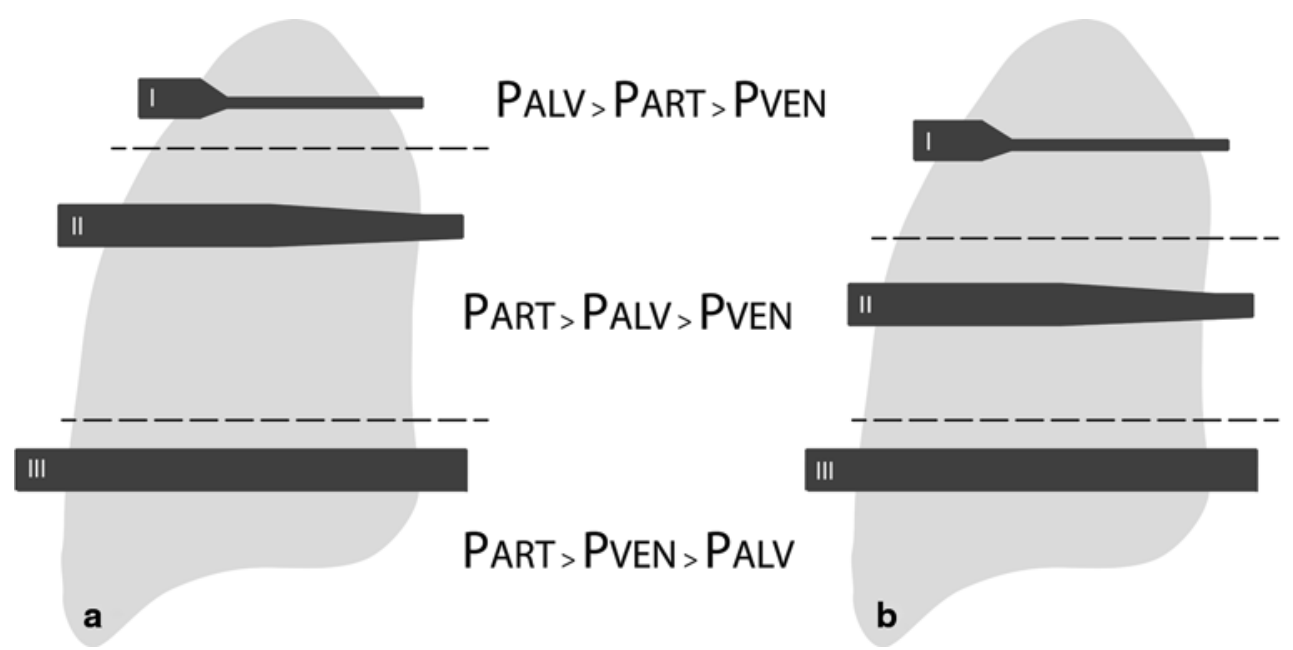

Fig. 2 A physiological model of the pulmonary vasculature in the upright position in a normal lung (a) versus a lung post-pneumonectomy (b). Due to gravitation, in the upright position blood flow in the apex of the lung is physiologically prevented since alveolar pressure exceeds the pulmonary arteriolar pressure (pulmonary zone I phenomenon). A high pulmonary vascular resistance in the post-pneumonectomy situation causes an increase in right ventricular afterload. When right ven-

to the patient's debilitated condition and severe hypoxaemia with $5 \mathrm{~L}$ oxygen, an urgent percutaneous PFO closure was performed with an $8 \mathrm{~mm}$ Amplatzer Septal Occluder. He recovered rapidly and was able to walk around the ward within 2 days with saturations of $97 \%$ in both supine and upright positions, after having only been able to lie in a supine position for the previous 3 weeks of his hospital stay. A TTE before hospital discharge showed no residual shunt. His follow-up after 4 months was uneventful with a nearnormal exercise capacity.

\section{Discussion}

We describe four cases with platypnoea-orthodeoxia syndrome caused by the combination of patent foramen ovale and a pulmonary condition with loss of pulmonary volume. The mechanism by which POS develops in the absence of pulmonary hypertension is often unclear. Two explanations have been postulated to explain POS in the presence of an inter-atrial communication: (1) a position-dependent transient pressure gradient across the inter-atrial septum and (2) an anatomical preferential blood flow through the interatrial communication [4-6].

Case 1, 2 and 4 had undergone pulmonary resection, in one patient this was left-sided and in two patients right-sided, after which they started developing dyspnoea. According to the first explanation, after pneumonectomy the right ventricular afterload increases and compliance decreases due to the reduced pulmonary vascular bed (Fig. 1a1, b1, d1) and the subsequent increase in pulmonary vascular resistance. tricular output reduces in the upright position, this afterload cannot be compensated. Consequently, pulmonary arteriolar pressure drops even more, causing a larger pulmonary zone I. I pulmonary zone I with restricted blood flow, II zone II with normal blood flow, III zone III with maximum blood flow; Palv alveolar pressure, Part arteriolar pressure, $P$ ven venous pressure

Right ventricular afterload is also increased by post-pneumonectomy fluid overload in the operated hemithorax [7, 8]. These haemodynamic changes cause a right-to-left shunt due to a transient pressure gradient since right atrial pressure increases. An upright position reduces right ventricular preload and cardiac output. Orthodeoxia might consequently develop due to an increase in the alveolar dead space known as 'pulmonary zone I' (Fig. 2), in which the pressure in alveoli exceeds the orthostatic decrease in pulmonary arteriolar pressures, causing an additional ventilation-perfusion mismatch [8]. Though not reported in our cases, a different cause of dyspnoea after lung surgery can be phrenic nerve injury, particularly after right-sided pneumonectomy [9, 10]. The elevated right hemidiaphragm causes right ventricular compression and subsequent outflow impairment, which in turn causes a transient pressure gradient resulting in the right-to-left shunt. In these particular cases, plication of the diaphragm is preferred over PFO closure to remove the underlying cause [9]. Figure 3 summarises the haemodynamic explanation in a schematic view.

The second explanation is the relocation of the atrial septum which facilitates preferential flow of caval venous blood into the opening of the atrial defect. Intrathoracic post-pneumonectomy changes also account for this positional change of the inter-atrial communication, along with the haemodynamic shift as described above. A spontaneous mediastinal relocation to the opposite side, which occurs approximately 3 weeks to 7 months after pneumonectomy, predisposes to stretching and distortion of the atrial septum as the inferior caval vein remains in place [11]. In the upright position additional stretching occurs due to gravity, which 


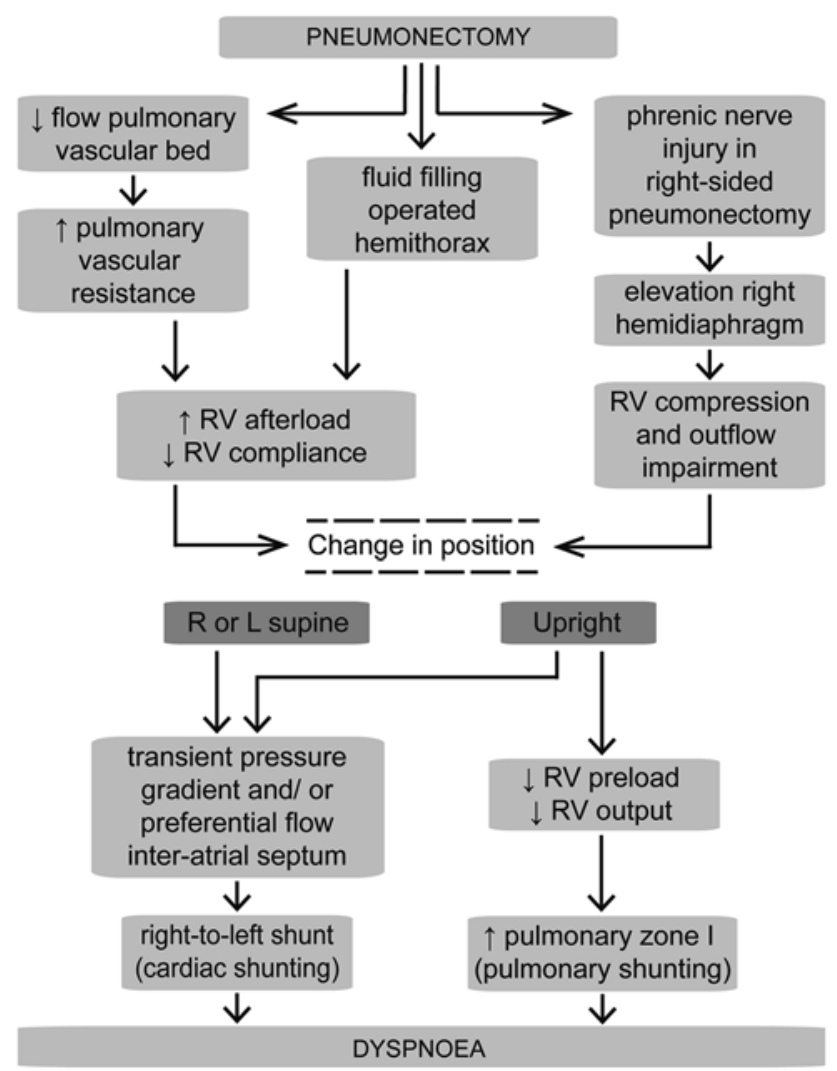

Fig. 3 Schematic view of the haemodynamic mechanism explaining the position-dependent dyspnoea after pneumonectomy in case of an inter-atrial communication. Through several pathways pneumonectomy can result in dyspnoea by a position-dependent transient pressure gradient or an anatomical preferential flow across the inter-atrial septum. In the upright position gravity leads to additional pulmonary shunting. $R V$ right ventricle, $R$ right, $L$ left

enlarges the inter-atrial communication and causes right-toleft shunting despite normal right atrial pressures $[3,4]$. In a review of reported POS cases, Marini et al. [12] noted a significantly higher prevalence of POS in post-pneumonectomy patients who developed respiratory symptoms within $6 \pm 7$ months compared with those with a symptomless interval of $<1$ month. Case 1, 2 and 4 indeed developed progressive dyspnoea within weeks to months after lung resection. The gradual synergic effect of atrial septal stretching along with a possible transient pressure gradient could explain the late onset of POS as seen in most reported cases [1, 6, 12].

Case 3 demonstrates POS caused by a PFO in a patient with elevated pulmonary artery pressure secondary to chronic pulmonary embolism. The haemodynamic mechanism involves elevated right atrial pressures due to relatively decreased right ventricular compliance, comparable with the post-pneumonectomy situation. Furthermore, in accordance with the preferential transseptal flow mechanism, she had a dilated aortic root and ascending aorta which in the upright position can horizontalise the atrial septum increasing caval venous flow through the defect, as well as compressing the right atrium to make the PFO more mobile and permeable for shunting [13-15].

Several important observations are worth mentioning. Firstly, in all presented cases POS was diagnosed several months after the onset of symptoms and clinical presentation. Beside the fact that clinicians are relatively unfamiliar with POS as compared with other causes of dyspnoea, its complicated underlying mechanism often requires different diagnostic imaging modalities which add to the delay in establishing a plausible diagnosis. Therefore clinicians must consider diagnosing POS when patients present with position-dependent dyspnoea, even before the common causes of dyspnoea have been excluded. Secondly, an immediate symptom relief is present as soon as the PFO is successfully closed, as confirmed in this case series. Clinical verification of the efficacy of PFO closure is indicated when symptom continuation implies a residual shunt.

In conclusion, diagnosing POS remains a challenge since clinicians must recognise the heterogeneous presentation in severely dyspnoeic patients. Position-dependent dyspnoea and desaturation should provide the clue. Initial assessment of POS can be made by observing the platypnoea, pulse oximetry and positional echocardiography. In some cases, POS can only be confirmed after observing the immediate symptom relief after PFO closure. Physicians should be reminded that a PFO, in combination with a pulmonary condition, can become symptomatic at older age as shown in this case series. In such cases, the debilitating dyspnoea can be successfully relieved by closure of the inter-atrial communication. Most importantly, clinical awareness is essential to recognise POS and prevent unnecessary delays.

\section{Funding None.}

\section{Conflict of interest None declared.}

Open Access This article is distributed under the terms of the Creative Commons Attribution License which permits any use, distribution, and reproduction in any medium, provided the original author(s) and the source are credited.

\section{References}

1. Knapper JT, Schultz J, Das G, Sperling LS. Cardiac platypnea-orthodeoxia syndrome: an often unrecognized malady. Clin Cardiol. 2014;37(10):645-9.

2. Burchell HB, Helmholz HFJ, Wood EH. Reflex orthostatic dyspnea associated with pulmonary hypertension. Am J Physiol. 1949;159:563-4.

3. Cheng TO. Mechanisms of platypnea-orthodeoxia: what causes water to flow uphill? Circulation. 2002;105(6):e47.

4. Rodrigues P, Palma P, Sousa-Pereira L. Platypnea-orthodeoxia syndrome in review: defining a new disease? Cardiology. 2012;123(1):15-23. 
5. Strunk BL, Cheitlin MD, Stulbarg MS, Schiller NB. Right-to-left interatrial shunting through a patent foramen ovale despite normal intracardiac pressures. Am J Cardiol. 1987;60(4):413-5.

6. Smeenk FW, Postmus PE. Interatrial right-to-left shunting developing after pulmonary resection in the absence of elevated right-sided heart pressures. Review of the literature. Chest. 1993;103(2):528-31.

7. Baumgartner F, Omari B, Lee J, et al. Survival after trauma pneumonectomy: the pathophysiologic balance of shock resuscitation with right heart failure. Am Surg. 1996;62(11):967-72.

8. Cheng TO. Platypnea-orthodeoxia syndrome: etiology, differential diagnosis, and management. Catheter Cardiovasc Interv. 1999;47(1):64-6.

9. Welvaart WN, Becker A, Marcus JT, et al. Unusual treatment of patent foramen ovale after pneumonectomy. Eur Respir J. 2010;35(4):929-31.
10. Aigner C, Lang G, Taghavi S, et al. Haemodynamic complications after pneumonectomy: atrial inflow obstruction and reopening of the foramen ovale. Eur J Cardiothorac Surg. 2008;33(2):268-71.

11. Christiansen KH, Morgan SW, Karich AF, Takaro T. Pleural Space Following Pneumonectomy. Ann Thorac Surg. 1965;122:298-304.

12. Marini C, Miniati M, Ambrosino N, et al. Dyspnoea and hypoxaemia after lung surgery: the role of interatrial right-to-left shunt. Eur Respir J. 2006;28(1):174-81.

13. Baptista R, da Silva AM, Castro G, et al. Ascending aortic aneurysm and patent foramen ovale: a rare cause of platypnea-orthodeoxia. Rev Port Cardiol. 2011;30(4):445-50.

14. Eicher JC, Bonniaud P, Baudouin N, et al. Hypoxaemia associated with an enlarged aortic root: a new syndrome? Heart. 2005;91(8):1030-5.

15. Buikema JW, van Geel PP. [Platypnea-orthodeoxia syndrome]. Ned Tijdschr Geneeskd. 2010;154:A2613. 